INPLASY

PROTOCOL

To cite: Liu et al. Comparison of efficacy and safety of traditional Chinese patent medicines for diabetic nephropathy: A Bayesian Network meta-analysis protocol. Inplasy protocol 202220114. doi:

10.37766/inplasy2022.2.0114

Received: 26 February 2022

Published: 26 February 2022

Corresponding author:

Shilin Liu

21102570121@stu.ccucm.edu.cn

Author Affiliation:

Changchun University of

Chinese Medicine.

Support: NKRDP

(No.2018YFC1704102).

Review Stage at time of this submission: Preliminary searches.

Conflicts of interest: None declared.

\section{Comparison of efficacy and safety of traditional Chinese patent medicines for diabetic nephropathy: A Bayesian Network meta-analysis protocol}

\author{
Liu, S1; Li, A2; Jiang, B3; Mi, J4; Nan, H5; Bao, P6; Nan, Z77.
}

Review question / Objective: This study will compare the efficacy and safety of different traditional Chinese patent medicines for diabetic nephropathy. The results of the study will provide a basis for the selection of adjuvant treatment options for diabetic nephropathy. We will search the major Chinese and English databases according to our search strategy. Search dates are from construction to 25 February 2022.According to this principle of participants, interventions, comparisons, outcomes (PICOS). To conduct a comprehensive and systematic search of the database of traditional Chinese patent medicine treatment diabetic nephropathy randomized controlled trial. The two researchers will use EndnoteX9 software to extract literature data and independently evaluate quality.Finally, the Bayesian network meta analysis is carried out by using software such as ReviewManager, stata16.0 and winbugs1.4.3.

INPLASY registration number: This protocol was registered with the International Platform of Registered Systematic Review and Meta-Analysis Protocols (INPLASY) on 26 February 2022 and was last updated on 26 February 2022 (registration number INPLASY202220114).

\section{INTRODUCTION}

Review question / Objective: This study will compare the efficacy and safety of different traditional Chinese patent medicines for diabetic nephropathy. The results of the study will provide a basis for the selection of adjuvant treatment options for diabetic nephropathy. We will search the major Chinese and English databases according to our search strategy.Search dates are from construction to 25 February 2022. According to this principle of participants, interventions, comparisons, outcomes 
(PICOS). To conduct a comprehensive and systematic search of the database of traditional Chinese patent medicine treatment diabetic nephropathy randomized controlled trial. The two researchers will use EndnoteX9 software to extract literature data and independently evaluate quality. Finally, the Bayesian network meta analysis is carried out by using software such as ReviewManager, stata16.0 and winbugs1.4.3.

Condition being studied: Background: Diabetic nephropathy is one of the most serious complications of diabetes.It has become a global public health problem for human health.Diabetic nephropathy is the leading cause of end-stage renal disease.At present, there is no specific medicine and therapy in modern medicine.In recent years, studies have shown that traditional Chinese patent medicines have been effective in treating diabetic nephropathy, with few side effects. There is no systematic review on the treatment of diabetic nephropathy with Chinese patent medicines.This study will systematically evaluate the efficacy and safety of Chinese patent medicines in the treatment of diabetic nephropathy. Methods: We will search the major Chinese and English databases according to our search strategy. Search dates are from construction to 25 February 2022. According to this principle of participants, interventions, comparisons, outcomes (PICO). To conduct a comprehensive and systematic search of the database of traditional Chinese patent medicine treatment diabetic nephropathy randomized controlled trial. The two researchers will use EndnoteX9 software to extract literature data and independently evaluate quality. Finally, the Bayesian network meta analysis is carried out by using software such as ReviewManager, stata16.0 and winbugs1.4.3. Results: This study will analyze the primary outcomes of Urine albumin excretion rate, Urea nitrogen, Serum creatinine, Total effective rate, and adverse events, and the secondary outcomes of Body mass index (BMI), Fasting blood glucose(FBG), 2hPGduring 75-g OGTT.To provide a reliable basis for the treatment of diabetic nephropathy with different traditional Chinese patent medicines. Conclusion: This study will compare the efficacy and safety of different traditional Chinese patent medicines for diabetic nephropathy. The results of the study will provide a basis for the selection of adjuvant treatment options for diabetic nephropathy.

\section{METHODS}

Participant or population: Select patients who meet the diagnostic criteria of diabetic nephropathy. The diagnostic criteria refer to the diagnostic criteria for diabetes issued by American Diabetes Association (ADA) in 2009 and Mogensen staging of diabetic nephropathy. There are no restrictions on gender, race, age, course of disease, TCM Syndrome.

Intervention: The experimental group was treated with traditional Chinese patent medicines on the basis of routine treatment.

Comparator: The control group was treated with western medicine on the basis of routine treatment.

Study designs to be included: Search dates are from construction to 25 February 2022. According to this principle of participants, interventions, comparisons, outcomes (PICOS). To conduct a comprehensive and systematic search of the database of traditional Chinese patent medicine treatment diabetic nephropathy randomized controlled trial. The two researchers will use EndnoteX9 software to extract literature data and independently evaluate quality. Finally, the Bayesian network meta analysis is carried out by using software such as ReviewManager, stata16.0 and winbugs1.4.3.

Eligibility criteria: 2.2. Inclusion criteria 2.2.1. Type of researchlncluded in the literature should be published at home and abroad traditional Chinese patent medicine treatment of diabetic nephropathy randomized controlled trial literature (RCT).Languages are limited to Chinese 
and English.Not Limited by time and whether or not to use the blind method.2.2.2. Types of patientsSelect patients who meet the diagnostic criteria of diabetic nephropathy. The diagnostic criteria refer to the diagnostic criteria for diabetes issued by American Diabetes Association(ADA) in 2009 and Mogensen staging of diabetic nephropathy. There are no restrictions on gender, race, age, course of disease, TCM Syndrome.2.2.3. InterventionsThe control group was treated with western medicine on the basis of routine treatment.The experimental group was treated with traditional Chinese patent medicines on the basis of routine treatment.The use of traditional Chinese patent medicines is limited to oral administration.The time, frequency and dosage of medication are not required.2.2.4. Outcomes1.Primary outcomes: Urine albumin excretion rate, Urea nitrogen, Serum creatinine, Total effective rate, and adverse events (e.g. hypoglycemia, gastroin-testinal symptoms, rash).2.Secondary outcomes:Body mass index (BMI), Fasting blood glucose(FBG), 2hPGduring 75-g OGTT, HbA1c, 24 hours urine protein quantification, Fasting insulin and 2-h postprandial insulin.2.3. Exclusion criteriaThe rules of exclusion are as follows: 1 . They are not diabetic nephropathy, or type 2 diabetes with Diabetic Ketoacidosis, urinary tract infections, severe heart, lung, or liver disease, or on dialysis.2.The experimental group was given other treatments besides traditional Chinese patent medicines.The control group was not treated with Western medicine. 3.The type of study is not a randomized controlled trial, such as animal studies, conference papers, reviews, case studies, and repeated literature.4.Literature without major outcome indicators or incomplete data reporting that can not be accessed.

Information sources: We have carried on the specialized training and the study to the literature retrieval method and the skill as well as the matters needing attention before the literature retrieval. After two prechecks, the retrieval strategy is finally formulated. The literature was incorporated into each database until February 25,2022. The searchable database includes eight databases, including PubMed, Embase, Cochrane Library, Web of Science, Chinese Biomedical Literature Database (CBM), Chinese National Knowledge Infrastructure (CNKI), Chinese Scientific Journal Database (VIP, Wanfang database. We will use the combination of subject words and free words, combined with the search specification of a specific database to formulate the search strategy.In addition, we will examine ongoing and unpublished studies registered with the World Health Organization's International Clinical Trial Registry. At the same time, we will manually search all reference lists from relevant system reviews for other eligible studies.The specific retrieval strategy for PubMed is shown in Table 1.The data will also be searched from other sources.

Main outcome(s): Urine albumin excretion rate, Urea nitrogen, Serum creatinine, Total effective rate, and adverse events (e.g. hypoglycemia, gastroin-testinal symptoms, rash).

Additional outcome(s): Body mass index (BMI), Fasting blood glucose(FBG), 2hPGduring 75-g OGTT, HbA1c, 24 hours urine protein quantification, Fasting insulin and 2-h postprandial insulin.

Quality assessment / Risk of bias analysis: 1.Risk of bias assessment - The two researchers independently evaluated the methodological quality of the studies using the Cochrane risk-bias assessment tool. The content of the evaluation includes the following six items: random sequence generation method; whether allocation concealment is used; whether the subject and the intervention provider are blinded; whether the result evaluator is blind; whether the result data is complete; Whether selective results reporting and other sources of bias.According to the relevant assessment criteria, the included studies will be rated as low risk of bias, high risk of bias and uncertainty of bias risk.If there are differences of opinion in the quality assessment, the decision is made in 
consultation with the third researcher until a consensus is reached. 2.Publication bias and evidence quality assessment - When analyzing the effect index, if the number of articles included is more than 10 , then the funnel chart is used to analyze the publication bias risk. When the funnel diagram is obviously asymmetric, it indicates that there is publication bias. The reliability of the evidence will be assessed through grading recommendations for evaluation, development and evaluation. The quality of the evidence will be classified as high, medium, low and very low.

Strategy of data synthesis: Data extraction - Two medical researchers (Shilin Liu and Andong Li) searched the literature based on a pre-determined search strategy. Using Endnote $\times 9$ software, the data were selected and extracted according to inclusion and exclusion criteria.The extracted data included the basic information of literature, the basic characteristics of patients, intervention measures, outcome indicators and so on.We will delete all meeting records, newspapers, guides, letters, and other literature, and to a unified standard for data extraction. The two researchers crosscheck, if there are different views, and third-party researchers (Zheng Nan) will be discussed in consultation, to make the final decision.When the full text or analysis of the literature found incomplete or missing information may affect the results of this study, we will attempt to contact the authors of the literature for data.The flow chart of the study is based on the PRISMA flow chart. This is shown in Figure 1. Statistical model selection - We will use Review Manager software(REVMAN v5.3 Cochrane Collaboration) and STATA16.0 software to perform a meta-analysis of the included literature. $\mathrm{P}<0.05$ was considered statistically significant.The two researchers will enter and account for the data independently and a third will review the data. The combined effects were represented by standardized mean square deviation(SMD) and $95 \%$ confidence interval $(95 \% \mathrm{Cl})$. The combination effect was expressed by ratio(OR) and $95 \% \mathrm{Cl}$.
Network meta-analysis - The network meta-analysis will use STATA16.0 software and introduce a random effects model to merge data and draw a network graph to show the direct and indirect comparison between different interventions.In the network, the bigger the arm, the bigger the basic data and the bigger the circle area, the better the effect of the intervention. Bayesian network meta-analysis is based on Markov-chain-Monte-Carlo(MCMC).We will use the MCMC in WinBUGS1.4.3 to analyze the random effects model with Bayesian mesh meta.Using 3 MCMC to simulate.Set the number of iterations to 100,000 and use the first 5,000 for annealing to eliminate the effect of the initial value.The consistency of each closed loop is evaluated by calculating the relative ratio (RoR) and its $95 \%$ confidence interval (CI). The lower limit of $95 \% \mathrm{Cl}$ equal to 1 indicates that the consistency is better.If the RoR is close to 1 , the direct evidence is consistent with the indirect evidence, and the fixed effect model is used for analysis. Otherwise, it is considered that there is obvious inconsistency in the closed loop, and the random effect model is used for analysis. The data of the two categories were expressed by $\mathrm{OR}$ and $95 \% \mathrm{Cl}$, and the difference was statistically significant.The curative effect of different intervention measures was ranked by WinBUGS1.4.3 software, and the area under the curve was recorded. The area under the curve is expressed as a percentage, and the larger the percentage, the better the therapeutic effect.

Subgroup analysis: If the results are not consistent, this study will be based on different reasons for the subgroup analysis. To explore the sources of Heterogeneity, including race, age, country, sex, dosage form, different forms of intervention and so on.

Sensitivity analysis: The Sensitivity analysis will be carried out by excluding every qualified document.After ruling out one study, If heterogeneity changes, the study may be a source of Heterogeneity.We will further analyse and explain why this document is a source of Heterogeneity.If 
the heterogeneity remains unchanged after exclusion of individual literature, the results of our study are relatively robust.

Country(ies) involved: China.

Keywords: Diabetic nephropathy, traditional Chinese patent medicine, safety, network meta-analysis, systematic review, protocol.

Contributions of each author:

Author 1 - Shilin Liu.

Author 2 - Andong Li.

Author 3 - Bin Jiang.

Author 4 - Jia Mi.

Author 5 - Hongmei Nan.

Author 6 - Pengjie Bao.

Author 7 - Zheng Nan. 